CHRONIC OBSTRUCTIVE PULMONARY DISEASE

\title{
Cost effectiveness of inhaled steroid withdrawal in outpatients with chronic obstructive pulmonary disease
}

\author{
J van der Palen, E Monninkhof, P van der Valk, S D Sullivan, D L Veenstra

See end of article for authors' affiliations

Correspondence to: Dr J van der Palen, Medisch Spectrum Twente, Concernstaf, Department of Epidemiology, P O Box $50000,7500 \mathrm{KA}$

Enschede, The Netherlands; vdpalen@ euronet.nl

Received 18 April 2005 Accepted

25 September 2005

Published Online First

21 October 2005
Background: The evidence for the effectiveness and safety of inhaled corticosteroids (ICS) in chronic obstructive pulmonary disease (COPD) is inconclusive. This study determined the cost effectiveness of withdrawing fluticasone propionate (FP) in outpatients with COPD.

Methods: The cost effectiveness analysis was based on a randomised, placebo controlled FP withdrawal study. After a 4 month run in period on FP, patients were randomly assigned to continue FP $500 \mu \mathrm{g}$ twice daily or to receive placebo for 6 months. A decision analytical model evaluated the 6 month incremental cost effectiveness of the ICS versus ICS withdrawal strategy. One way sensitivity analyses and a Monte Carlo simulation were performed to evaluate the robustness of the findings.

Results: The average patient with COPD in the FP group generated $€ 511$ in direct medical costs, including $€ 238$ for FP. The cost of the placebo strategy was $€ 456$. The higher direct drug cost of $€ 212$ per patient for the FP strategy during the 6 month follow up period compared with the placebo group was partially offset by a lower exacerbation and hospital admission cost of $€ 157$. The 6 month incremental cost effectiveness of the FP strategy compared with placebo was $€ 110$ per exacerbation prevented and $€ 1286$ per hospital admission prevented.

Conclusions: Over a 6 month period, withdrawing FP in a pre-selected trial population of COPD patients led to absolute cost savings but with a higher rate of exacerbations and hospital admissions.

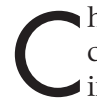
ronic obstructive pulmonary disease (COPD) is common disease that affects up to 24 million people death. Patients with COPD have about three exacerbations of their disease per year, many of which result in unscheduled visits to a physician or emergency department and to admission to hospital. Clinicians regularly prescribe long term inhaled corticosteroids (ICS) for the management of COPD, as ICS are effective in controlling inflammation in asthma and both asthma and COPD result from chronic inflammation. However, the inflammatory pattern of COPD is different from that of asthma, and evidence for the effectiveness and safety of ICS in COPD is contradictory. ${ }^{1-5}$ Recent meta-analyses also show conflicting results on the ability of ICS to arrest the long term decline in forced expiratory volume in 1 second $\left(\mathrm{FEV}_{1}\right)$ in COPD and its effects on symptoms and numbers of exacerbations. Trade offs between potential clinical benefits and harms remain unclear. ${ }^{67}$ However, the GOLD guidelines on COPD recommend that ICS should be prescribed in patients with an FEV $_{1}$ of $<50 \%$ predicted and frequent exacerbations. ${ }^{8}$

Only four studies have investigated the effect of withdrawal of ICS in COPD: an observational non-randomised study as part of the run in phase of the ISOLDE study; ${ }^{9}$ a small underpowered crossover study with a short follow up and no washout period; ${ }^{10}$ our own study (the COPE study); $i^{11}$ and, recently, the COSMIC study. ${ }^{12}$ In the run in phase of the ISOLDE study ICS were withheld from patients already using these medications. In the first 7 weeks after withdrawal 38\% of patients previously treated with ICS experienced an exacerbation compared with $6 \%$ of those who had not previously received ICS. Similarly, in the COPE study the majority of exacerbations also occurred in the first 7 weeks. The crossover study by $\mathrm{O}^{\prime}$ Brien $e t \mathrm{al}^{10}$ showed that withdrawal of ICS in elderly patients with COPD led to deterioration in ventilatory function and increased exercise induced dyspnoea with a trend towards an increased frequency of exacerbations. However, the results of this small crossover study should be viewed with caution as only 15 of the 24 patients completed the study and follow up was only 12 weeks. The clinical results of the COPE study are published in detail elsewhere. ${ }^{11}$ In brief, after 4 months of treatment with FP $(1000 \mathrm{mg} /$ day $), 244$ patients were randomised to either continue $\mathrm{FP}$ or to receive placebo for 6 months. In the placebo group 26 patients (21.5\%) experienced rapid recurrent exacerbations and were subsequently unblinded and prescribed open FP compared with six patients $(4.9 \%)$ in the FP group (relative risk $(\mathrm{RR})=4.4 ; 95 \%$ CI 1.9 to 10.3 ). With regard to the effect of ICS withdrawal on exacerbations, the COSMIC study showed a doubling of the incidence rate of mild exacerbations, but not moderate to severe exacerbations, in the year after withdrawing ICS in COPD patients also using salmeterol. ${ }^{12}$

The objective of the current economic evaluation was to assess the cost effectiveness of withdrawing the ICS fluticasone propionate (FP) $500 \mu \mathrm{g}$ twice daily in outpatients with COPD based on the 6 months of follow up in the COPE study. We used decision modelling techniques to assess differences in total costs as well as the cost per exacerbation and per hospital admission prevented over the 6 month trial period. Modelling allowed us to explore variation in parameters to enhance generalisability.

\section{METHODS}

Clinical data

The COPE study was a randomised, double blind, parallel group, single centre study with a 4 month run in period and

Abbreviations: COPD, chronic obstructive pulmonary disease; FP, fluticasone propionate; ICS, inhaled corticosteroids 


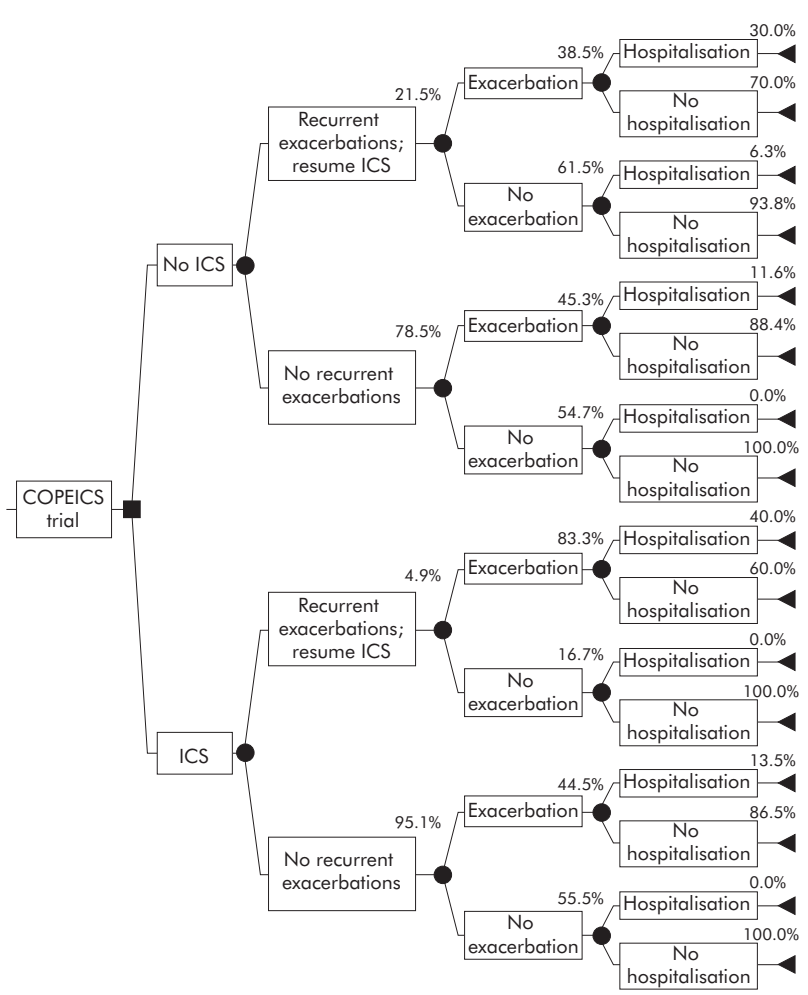

Figure 1 Decision analytical model with probablilities of exacerbations and hospitalisations for the inhaled corticosteroid (ICS) strategy and the placebo strategy.

6 months of active treatment or placebo, with follow up visits at 3 and 6 months. The inclusion criteria and design have been described previously and are summarised briefly below. ${ }^{11}$ In the run in phase all patients were prescribed FP via Diskus $500 \mu \mathrm{g}$ twice daily to optimise lung function. After the 4 month run in period, eligible patients were randomly assigned to continue FP $500 \mu \mathrm{g}$ twice daily or to receive placebo for 6 months. If patients experienced any worsening of their respiratory symptoms they were invited to attend the hospital within 12 hours for spirometry measurements and consultation by one of the study physicians who subsequently decided either to continue the trial or to prescribe FP $500 \mu \mathrm{g}$ twice daily unblinded. The latter was allowed according to the "benefit of the doubt" principle in case patients experienced rapid recurrent exacerbations. This was defined as either twice an objective increase in respiratory symptoms within a 3 month period (defined as a decrease in
$\mathrm{FEV}_{1}$ of more than $20 \%$ or $300 \mathrm{ml}$ compared with stable lung function at randomisation) or three times a subjective increase in respiratory symptoms in a 3 month period as experienced by the patient regardless of the abovementioned criteria. For patients who were already on FP, treatment therefore did not change; only the blinding was gone.

The study was approved by the Medical Ethical Committee of Medisch Spectrum Twente, Enschede, The Netherlands. All patients in the COPE study gave informed consent.

\section{Economic evaluation using a decision analytical model}

A decision analytical model with a time perspective of 6 months was developed to evaluate the short term (incremental) cost effectiveness of the ICS versus withdrawal strategy. Figure 1 depicts the decision analytical model. Table 1 presents the base case probabilities with the associated 95\% confidence intervals (95\% CI) for each step in the model. All data come from the COPE study.

Base case cost effectiveness analyses were performed according to the US panel on cost effectiveness analysis guidelines. ${ }^{13-15}$ However, indirect costs such as lost productivity during usual daily activities were excluded from the base case analyses, thus assuming the perspective of the healthcare payer. The cost effectiveness ratio was calculated as cost per exacerbation prevented and cost per hospital admission prevented, respectively. One way sensitivity analyses were performed to evaluate the relative impact of the various parameters in the decision analytical model. Cost components with the exception of hospital costs were varied over a range of $50 \%$ to $150 \%$ of the actual cost. The probabilities of experiencing exacerbations or hospital admissions and the costs associated with hospital admissions were varied between the lower and upper 95\% confidence intervals (CI) derived from the COPE trial data. A Monte Carlo simulation with 1000 iterations was performed to explore the variation in the total costs as well as the cost per exacerbation and hospital admission prevented when cost parameters and probabilities were varied simultaneously over their ranges and associated 95\% CI. For the cost of exacerbations and FP, triangular distributions were used. The reason for varying the cost of FP in the model was to facilitate generalisation to situations where ICS are cheaper or more expensive. For the cost of a hospital admission a normal distribution was used, while a logistic normal distribution was used for all probabilities. ${ }^{16}$

\section{Resources and costs}

Healthcare resource use was prospectively recorded during the COPE study by active follow up of the patients' records

Table 1 Base case values of probabilities of exacerbations and hospital admissions

\begin{tabular}{lll}
\hline & Base case value $(95 \% \mathrm{Cl})$ & \\
\cline { 2 - 3 } & FP & Placebo \\
\hline $\begin{array}{l}\text { Probability of rapid recurrent exacerbations } \\
\text { Probability of further exacerbations when returned } \\
\text { to open FP }\end{array}$ & $0.049(0.023$ to 0.102$)$ & $0.215(0.151$ to 0.296$)$ \\
$\begin{array}{l}\text { Probability of at least one hospital admission in } \\
\text { patients who continue to experience exacerbations }\end{array}$ & $0.400(0.118$ to 0.769$)$ & $0.300(0.108$ to 0.603$)$ \\
$\begin{array}{l}\text { following use of open FP } \\
\text { Probability of at least one hospital admission during }\end{array}$ & $0.000(0.000$ to 0.793$)$ & $0.063(0.224$ to 0.575$)$ \\
$\begin{array}{l}\text { the } 6 \text { month trial period in patients free of } \\
\text { exacerbations following use of open FP }\end{array}$ & 0.011 to 0.283$)$ \\
$\begin{array}{l}\text { Probability of at least one exacerbation in patients } \\
\text { not experiencing recurrent exacerbations }\end{array}$ & $0.445(0.358$ to 0.535$)$ & $0.453(0.356$ to 0.553$)$ \\
$\begin{array}{l}\text { Probability of at least one hospital admission in } \\
\text { patients not experiencing recurrent exacerbations }\end{array}$ & $0.135(0.067$ to 0.253$)$ & $0.116(0.051$ to 0.245$)$ \\
\hline FP, fluticasone propionate. & & \\
\hline
\end{tabular}


Table 2 Six month cost $(€)$ and effects of withdrawal of fluticasone propionate (FP) $500 \mu \mathrm{g}$ twice daily versus placebo in outpatients with COPD using 2002 cost prices

\begin{tabular}{|c|c|c|c|}
\hline & FP strategy & Placebo strategy & Difference \\
\hline \multicolumn{4}{|l|}{ Cost per patient } \\
\hline FP $500 \mu \mathrm{g}$ twice daily* & 238 & 26 & 212 \\
\hline Exacerbation cosit & 59 & 93 & -34 \\
\hline Hospital admission $\ddagger$ & 214 & 337 & -123 \\
\hline Total direct medical cost & 511 & 456 & 55 \\
\hline \multicolumn{4}{|l|}{ Effect per patient } \\
\hline No of exacerbations & 0.87 & 1.37 & -0.50 \\
\hline No of hospital admissions & 0.073 & 0.116 & -0.043 \\
\hline \multicolumn{4}{|c|}{$\begin{array}{l}\text { *Includes } € 6 \text { pharmacy cost/prescription. } \\
\text { tIncludes salary cost of pulmonary physician and lung function assistant, as well as cost for courses of oral steroids } \\
\text { and antibiotics including } € 6 \text { pharmacy cost/prescription. } \\
\ddagger \text { Mean of } 10.5 \text { hospital days. }\end{array}$} \\
\hline
\end{tabular}

(both inpatients and outpatients) with regard to hospital admissions, emergency room visits, and scheduled and emergency outpatient visits. At each visit patients were questioned about possible adverse events and healthcare contacts. We also contacted all the patients' general practitioners to enquire about treated exacerbations of COPD at the end of the 6 month follow up period. Pharmacists reported all drugs used during the study period.

Current Dutch guidelines on good pharmacoeconomic practice specify that costs estimated at a national average level should be used as much as possible. ${ }^{17}$ Resource use, including the salary of the pulmonary physicians and lung function technicians, was multiplied by 2002 unit prices. ${ }^{18} 19$ Medication costs for FP, prednisolone, and amoxicillin/ clavulanate were based on market prices and included a €6 dispensing fee added for each 6 month period. During the trial $21.5 \%$ of patients in the placebo group experienced recurrent exacerbations and they resumed open FP treatment for the remainder of the trial. On average they used FP for $50 \%$ (91 days) of the entire trial period of 6 months.

Where applicable, Dutch guilders were converted into euros ( $1 €=$ NLG 2.20). For conversion to US dollars, costs in euros should be multiplied by a factor of 0.934 , based on the 2002 Purchasing Power Parities as issued by the Organisation for Economic Cooperation and Development (www.oecd.org). Because of the short time perspective, costs and effects were not discounted for time preferences.

\section{RESULTS}

\section{Base case cost effectiveness analysis of the trial}

The 6 month cost and effect data are presented in table 2. The average patient with COPD in the FP group generated $€ 511$

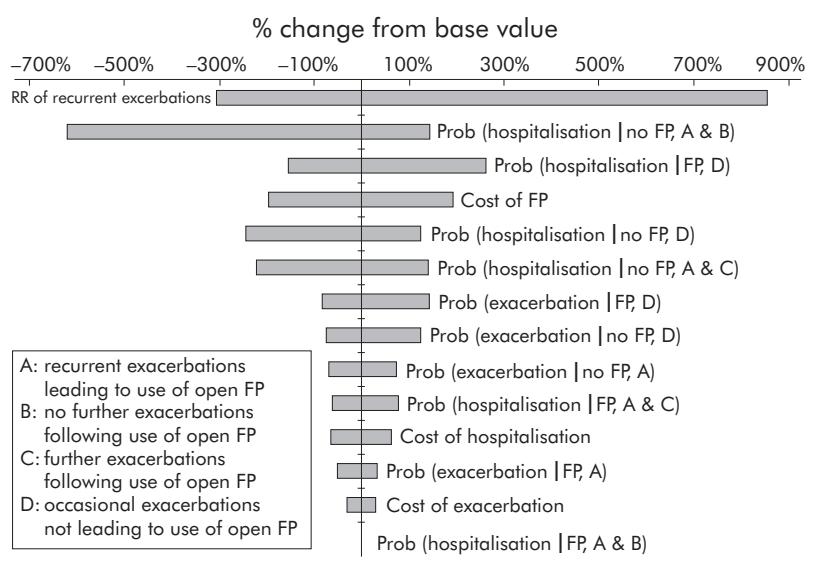

Figure 2 Tornado diagram for cost per exacerbation prevented. A positive change from base value favours the placebo strategy. in direct medical costs including $€ 238$ for FP. The cost of the placebo strategy was $€ 456$. The higher direct drug cost in the FP group of $€ 212$ per patient in the 6 month follow up period compared with the placebo group was partially offset by a lower exacerbation and hospitalisation cost of $€ 157$.

In the base case cost effectiveness analysis the 6 month incremental cost effectiveness of the FP strategy compared with placebo was $€ 110$ per exacerbation prevented and $€ 1286$ per hospital admission prevented. The corresponding Number Needed to Treat (NNT) to prevent one exacerbation is 2, while the NNT to prevent one hospital admission is 24 .

\section{Sensitivity analysis of the decision analytical model}

The results from the cost effectiveness analysis with regard to cost per exacerbation and hospital admission prevented were sensitive to changes in various parameters (fig 2). The Tornado diagram shows how the main outcome parameter (cost per exacerbation prevented) varies when the various inputs in the decision tree (probabilities, RR of recurrent exacerbations, and costs) are varied according to their distributions (normal and logistic normal distributions with their associated 95\% CI for hospital costs and probabilities mentioned in table 1 , as well as the RR, respectively; triangular distributions for the cost of exacerbations and FP).

When the RR of recurrent exacerbations following FP withdrawal (RR observed in the COPE study $=4.4$ ) decreases to the lower limit of the $95 \% \mathrm{CI}(\mathrm{RR}=1.9)$, the FP strategy exceeds a cost of $€ 1000$ per exacerbation prevented. At an RR of 5.4, both alternatives are equally costly. The same is true if the cost of FP is reduced to $75 \%$ of the base case cost at $€ 177$ per 6 months. The results are also sensitive to the probability of a hospital admission in those who develop recurrent

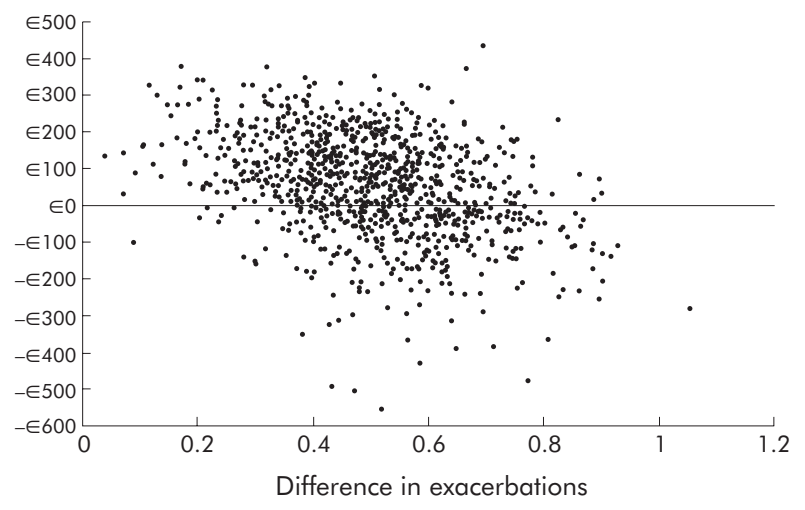

Figure 3 Results of a Monte Carlo simulation on cost per exacerbation prevented. A positive $€$ amount favours the placebo strategy and a positive difference in the number of exacerbations favours the FP strategy. 


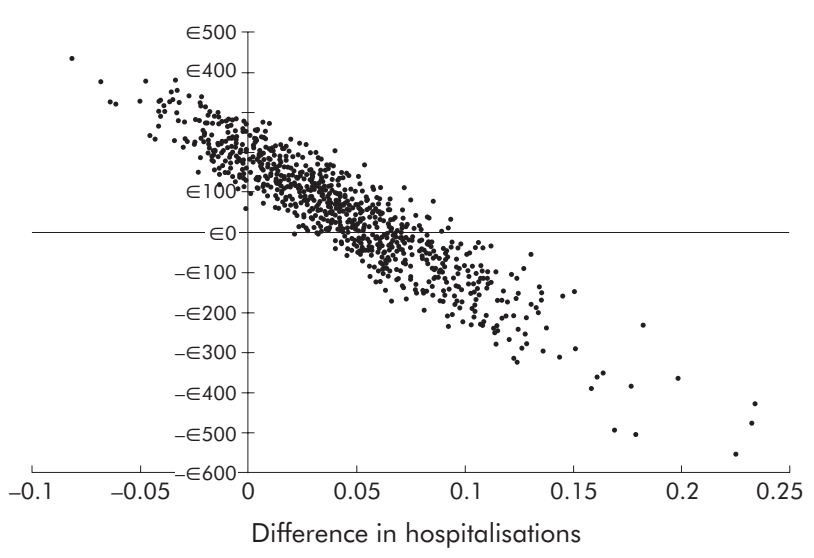

Figure 4 Results of a Monte Carlo simulation on cost per hospital admission prevented. A positive $€$ amount favours the placebo strategy and a positive difference in the number of exacerbations favours the FP strategy.

exacerbations following FP withdrawal but remain without exacerbations following use of open FP. At the upper limit of the $95 \%$ CI, with a probability of a hospital admission of $28 \%$, the FP strategy would save $€ 571$ per exacerbation prevented. Furthermore, the results are sensitive-but to a lesser degree-to the probability of a hospital admission in those who develop recurrent exacerbations following FP withdrawal and continue to have subsequent exacerbations following use of open FP (range of cost per exacerbation prevented from $€ 264$ to a saving of $€ 134$ ) and in those who only have an occasional exacerbation following FP withdrawal (range of cost per exacerbation prevented from €245 to a saving of $€ 157$ ). Finally, the results are sensitive to the probability of a hospital admission in those who remain on FP and only have an occasional exacerbation (range of cost per exacerbation prevented from $€ 402$ to a saving of $€ 57$ ).

A Monte Carlo simulation with 1000 iterations reached convergence. The median cost per exacerbation prevented was $€ 127$ (interquartile range $-€ 52$ to $€ 331$; fig 3 ) and the median cost per hospital admission prevented was $€ 122$ (interquartile range $-€ 1411$ to $€ 3069$; fig 4).

\section{DISCUSSION}

In the base case cost effectiveness analysis the 6 month incremental cost effectiveness of the FP strategy compared with placebo was $€ 110$ per exacerbation prevented and $€ 1286$ per hospital admission prevented. However, sensitivity analyses showed that the short term results are sensitive to the risk of recurrent exacerbations when withdrawing FP. The COPE study showed that only a minority of patients will develop these recurrent exacerbations following ICS withdrawal. ${ }^{11}$ The recently published COSMIC study showed a doubling of the incidence rate of mild exacerbations, but not moderate to severe exacerbations, in the year after withdrawing ICS in COPD patients also using salmeterol. ${ }^{12}$ Neither study provides evidence of a great danger of at least trying the withdrawal strategy. Also, these two studies considered exacerbations when ICS are stopped and do not provide evidence about whether the incidence rate of exacerbations will be reduced when ICS are started.

When the risk of exacerbations with the withdrawal of FP becomes relatively low, the FP strategy becomes very expensive. Given the sensitivity of the cost effectiveness ratio to the risk of recurrent exacerbations when withdrawing FP, it is important to identify patients with COPD in whom withdrawing FP is likely to be safe or not. The GOLD guidelines on COPD recommend that ICS should be prescribed in patients with an $\mathrm{FEV}_{1}$ of $<50 \%$ predicted and frequent exacerbations. ${ }^{8}$ Analysis of the subgroup of patients with an $\mathrm{FEV}_{1}$ of $<50 \%$ predicted in the COPD ICS withdrawal study suggested that the difference in time to first exacerbation between groups was driven by this subset. ${ }^{11}$

The results were also sensitive to the cost of FP. The sensitivity analysis of the trial results shows that the FP strategy becomes very expensive if the cost of FP per patient is doubled. This can be a result of doubling the dose of FP, although this is not very realistic as the patients in the study were already receiving $1000 \mu \mathrm{g}$ per day which is considered to be the maximum dose for maintenance therapy. The cost of FP can also be doubled when the drug is twice as expensive, which could be the case in other countries. If the cost of FP is reduced to below $75 \%$, the FP strategy becomes dominant. If this reduction in monthly cost is achieved with the same dosage of $1000 \mu \mathrm{g}$ per day, this holds true.

The unfavourable effects of withdrawing FP manifest themselves at an early stage. In patients who were returned to open FP, the average time it took to develop two objective exacerbations was exactly 3 months from the moment FP was withdrawn. When patients are returned to FP, it can be argued that they will have a similar future risk of adverse events as those who remained on FP. A long term study of the effects of withdrawing ICS in all COPD patients and resuming ICS only in those with rapid recurrent exacerbations might shed light on this matter.

In summary, withdrawing FP in a pre-selected trial population of patients with COPD led to absolute cost savings but with a higher rate of exacerbations and hospital admissions. In the long term, however, withdrawal of ICS from patients on long term ICS treatment and close follow up to see if they deteriorate in the first few months might be an appropriate strategy. Treatment with ICS should be resumed in those who have rapid recurrent exacerbations following withdrawal. Pre-screening of patients (for example, those without asthmatic features) is highly recommended, both to prevent unnecessary harm to patients and to prevent an unnecessarily high workload for the physician.

\section{Authors' affiliations \\ J van der Palen, E Monninkhof, P van der Valk, Department of Pulmonary Medicine, Medisch Spectrum Twente, Enschede, The Netherlands \\ S D Sullivan, D L Veenstra, Department of Pharmacy, University of Washington, Seattle, USA}

Source(s) of support in the form of grants: The Netherlands Asthma Foundation, Boehringer Ingelheim, Amicon Health Care Insurance Company, and GlaxoSmithKline BV. Source of support in the form of drugs and placebos: GlaxoSmithKline BV.

Competing interests: none.

No party had any role in the design of the study, the collecting of data, the analysis and interpretation of the data, and in the preparation, approval, and submission of the manuscript.

\section{REFERENCES}

1 Lipworth BJ. Systemic adverse effects of inhaled corticosteroid therapy: a systematic review and meta-analysis. Arch Intern Med 1999;159:941-55.

2 Calverley PM. Inhaled corticosteroids are beneficial in chronic obstructive pulmonary disease. Am J Respir Crit Care Med 2000;161:341-2.

3 Barnes PJ. Chronic obstructive pulmonary disease. N Engl J Med 2000;343:269-80.

4 Barnes PJ. Inhaled corticosteroids are not beneficial in chronic obstructive pulmonary disease. Am J Respir Crit Care Med 2000;161:342-4.

5 Keatings VM, Jatakanon A, Worsdell YM, et al. Effects of inhaled and oral glucocorticoids on inflammatory indices in asthma and COPD. Am J Respir Crit Care Med 1997; 155:542-8.

6 Highland KB, Strange C, Heffner JE. Long-term effects of inhaled corticosteroids on $\mathrm{FEV}_{1}$ in patients with chronic obstructive pulmonary disease. A meta-analysis. Ann Intern Med 2003;138:969-73.

7 Sin DD, McAlister FA, Man SF, et al. Contemporary management of chronic obstructive pulmonary disease: scientific review. JAMA 2003;290:2301-12. 
8 Pauwels RA, Buist AS, Calverley PM, et al. Global strategy for the diagnosis, management, and prevention of chronic obstructive pulmonary disease. NHLBI/WHO Global Initiative for Chronic Obstructive Lung Disease (GOLD) Workshop summary. Am J Respir Crit Care Med 2001:163:1256-76.

9 Jarad NA, Wedzicha JA, Burge PS, et al. An observational study of inhaled corticosteroid withdrawal in stable chronic obstructive pulmonary disease. ISOLDE Study Group. Respir Med 1999;93:161-6.

10 O'Brien A, Russo-Magno P, Karki A, et al. Effects of withdrawal of inhaled steroids in men with severe irreversible airflow obstruction. Am J Respir Crit Care Med 2001; 164:365-71.

11 van der Valk PD, Monninkhof E, Van der Palen J, et al. Effect of discontinuation of inhaled corticosteroids in patients with chronic obstructive pulmonary disease: the COPE study. Am J Respir Crit Care Med 2002; 166:1358-63.

12 Wouters EF, Postma DS, Fokkens B, et al. Withdrawal of fluticasone propionate from combined salmeterol/fluticasone treatment in patients with COPD causes immediate and sustained disease deterioration: a randomised controlled trial. Thorax 2005;60:480-7.
13 Weinstein MC, Siegel JE, Gold MR, et al. Recommendations of the Panel on Cost-Effectiveness in Health and Medicine. JAMA 1996;276:1253-8.

14 Siegel JE, Weinstein MC, Russell LB, et al. Recommendations for reporting cost-effectiveness analyses. Panel on Cost-Effectiveness in Health and Medicine. JAMA 1996:276:1339-41.

15 Russell LB, Gold MR, Siegel JE, et al. The role of cost-effectiveness analysis in health and medicine. Panel on Cost-Effectiveness in Health and Medicine. JAMA 1996;276:1172-7.

16 Doubilet $P$, Begg $C B$, Weinstein $M C$, et al. Probabilistic sensitivity analysis using Monte Carlo simulation. A practical approach. Med Decis Making 1985;5:157-77.

17 Oostenbrink JB, Koopmanschap MA, Rutten FF. Handleiding voor Kostenonderzoek; methoden en richtlijnprijzen voor economische evaluatie in de gezondheidszorg. Amstelveen: College voor Zorgverzekeringen, 2000.

18 Tariefboek Medisch Specialisten. Euroboek per 1 April 2002. Utrecht: College Tarieven Gezondheidszorg (CTG), 2002.

19 Oostenbrink JB, Koopmanschap MA, Rutten FF. Standardisation of costs: the Dutch Manual for Costing in economic evaluations. Pharmacoeconomics 2002;20:443-54.

\section{LUNG ALERT}

Tumour suppressor gene methylation and screening for lung cancer

$\Delta$ Belinsky SA, Klinge DM, Dekker JD, et al. Gene promoter methylation in plasma and sputum increases with lung cancer risk. Clin Cancer Res 2005;11:6505-11

B ronchial carcinoma is the leading cause of cancer death and often presents at an advanced stage. This study explored the exciting possibility of developing a screening tool for lung cancer. It assessed the level of inhibitory methylation of specific tumour suppressor genes in three groups of women with different levels of lung cancer risk. Plasma and sputum samples were taken from 74 never smokers (lowest risk with $<1 \times 10^{-4}$ lifetime risk), 121 current and former smokers (intermediate risk with $0.3 \%$ yearly risk), and 56 patients who had undergone surgical resection of stage I lung cancer (highest risk with $6.0 \%$ risk per patient year). Once DNA had been extracted from the samples, methylation specific polymerase chain reaction (PCR) was used to detect inhibition of specific genes: CDKN2A (pl6), $\mathrm{O}^{6}$-methylguanine-DNA methyltransferase (MGMT), and ras effector homologue 1 (RASSF1A) in plasma and sputum, with additional genes in sputum.

The prevalence of methylation was found to be greater in those with an increased cancer risk. In plasma the largest difference between the groups was seen for MGMT and pl6 where lung cancer survivors had increased odds of $5.0(p=0.07)$ and $3.2(p=0.04)$ fold for methylation compared with never smokers. Those with the highest risk were also more likely to have at least one gene affected $(\mathrm{p}<0.01)$. In sputum, lung cancer survivors had a 6.2 fold greater odds for methylation of three or more genes than smokers. Levels of RASSFiA and MGMT methylation in lung cancer survivors were significantly higher in sputum than in plasma samples $(\mathrm{p}<0.05)$. The authors conclude that the methylation of multiple gene promoters in sputum is strongly associated with the increased risk of lung cancer.

These results suggest that, with further work, the detection of gene methylation in sputum could be developed as a screening test for lung cancer. Larger studies evaluating this technique are urgently required.

R Stretton

Senior House Officer, Aberdeen Royal Infirmary, UK; r.stretton@nhs.net 